\title{
Uncertainty and the Allocation of Resources*
}

\author{
Harris Dellas ${ }^{\dagger} \quad$ Ana Fernandes ${ }^{\ddagger}$
}

October 26, 2009

\begin{abstract}
We study the effects of uncertainty on the allocation of resources in the standard, general equilibrium, two-sector, two-factor model. The elasticity of substitution in consumption plays the key role in determining whether uncertainty attracts or repels resources, while risk aversion and the production structure are of lesser importance. The model predicts that countries with lower consumption flexibility (poorer substitution possibilities) will be able to pursue more risky activities than economies with greater flexibility.
\end{abstract}

JEL class: E2, D5, D8

Keywords: Uncertainty, general equilibrium, two factor-sector model, flexibility

${ }^{*}$ We would like to thank Marios Angeletos, Michele Boldrin, Ron Jones, Bob Lucas, Sergio Rebelo and Anne Sibert for useful conversations.

${ }^{\dagger}$ Department of Economics, University of Bern, CEPR. Corresponding author: VWI, Schanzeneckstrasse 1, CH-3012 Bern, Switzerland. Tel: +41(0)31-631-3989, email: harris.dellas@vwi.unibe.ch, http://staff.vwi.unibe.ch/dellas/

${ }_{\ddagger}^{\ddagger}$ Department of Economics, University of Bern, email: ana.fernandes@vwi.unibe.ch, http://anafernandes name 


\section{Introduction}

Does uncertainty attract or repel resources? This is one of the most important questions in human affairs. In economics, it has been studied in a wide variety of contexts, including portfolio theory, savings-consumption choices, human capital and occupational decisions, economic development, growth and so on. Remarkably, this question has not been addressed in the context of the most natural -for this question- and also most empirically relevant economic model. Namely, the general equilibrium, multisector, multifactor neoclassical economy where different sectors (and/or inputs) are subject to different degrees of economic uncertainty. In such an environment, the standard elements identified in the literature (such as the degree of risk aversion) may fail to provide a complete or even accurate picture of how uncertainty affects the allocation of resources given the prominent role enjoyed by other parameters, namely, the elasticity of substitution among inputs in production or among goods in consumption.

Admittedly, there exist two distinct literatures that have dealt with uncertainty in general equilibrium, multisector economies. Namely, the theory of international trade under uncertainty (Helpman and Razin (1978)), and the real business cycle theory (Kydland and Prescott (1982), King and Rebelo (2000)). Nonetheless, neither of these literatures has contributed sufficiently to this issue.

The international trade literature has been exclusively concerned with the questions of (a) whether the presence of uncertainty undermines comparative advantage as the basis for trade and, (b) whether and under what conditions the standard propositions of trade (HeckscherOhlin - H-O- theorem, international factor price equalization, and so on) still hold under uncertainty. These questions are undoubtedly important but are only partly related to the issue of how uncertainty affects the allocation of resources. For instance, a finding that the $\mathrm{H}-\mathrm{O}$ theorem still holds under uncertainty does not suffice to determine the direction in which production shifts as a result of uncertainty. ${ }^{1}$

The real business cycle literature, on the other hand, has relied on models with certainty equivalence (typically by using a linear approximation around the deterministic steady state). Thus, by construction, it has shunned away from dealing with the effects of uncertainty on average (long term) allocations.

The work that has got the furthest along this front is that by Rothemberg and Smith (1970),

\footnotetext{
${ }^{1}$ An additional weakness of the trade literature is that it has only managed to address special cases of uncertainty.
} 
who use a static, two-factor, two-good model. However, in spite of the pioneering nature and importance of their work, their approach assumes that prices are given and hence, like standard portfolio models, it remains partial equilibrium.

Our paper thus represents the first attempt in the literature to study the general equilibrium ${ }^{2}$ effects of uncertainty in a $2 X 2$ model. The first important result obtained is that risk aversion is not necessary for uncertainty to influence economic decisions. Namely, uncertainty matters even under risk neutrality. A second result is that the key determinant of the relationship between uncertainty and the allocation of resources is the ease (flexibility) with which consumers are willing to substitute among consumption goods. When this is low, higher uncertainty is likely to attract resources, in particular when that sector's share in GDP is small (less then $50 \%$ in the two good case considered here). Thus, an important implication of the analysis is that countries that possess limited possibilities for substitution in consumption will choose to undertake more risky activities. To the extent that such activities carry higher returns, consumption inflexibility may support higher income (or growth). These findings indicate that it may not be possible to fully understand the effects of uncertainty on the level and growth of economic activity unless the degree of consumption flexibility and the structure of production are taken into account.

Our analysis may be useful for other literatures. For instance, it could help generalize standard portfolio analysis to deal with situations with endogenous rates of return or when assets returns are imperfect substitutes from the point of view of consumption. This is relevant when the object of study is an entire country rather than an individual investor. Similarly, it could be used to study the optimal degree of production diversification for firms that operate and also have market power in more than one sector. It could also be applied not only to economic growth and the determination of long term economic performance but also to issues pertaining to the long term effects of macroeconomic stabilization policies.

\section{The model}

The model is the standard, static, perfectly competitive, closed economy, two-good, twofactor model, with a representative agent and complete asset markets.

\footnotetext{
${ }^{2}$ Obstfeld's (1994) general equilibrium multi-country model can be interpreted as a multi-sector one. But, as in standard portfolio theory, there is no production in that model, there are no consumption substitution possibilities and the rates of return are exogenously given.
} 
Utility is derived from consumption of two goods, $X$ and $Y$, in amounts $c_{x}$ and $c_{y}$ respectively:

$$
U=U\left(C\left(c_{x}, c_{y}\right)\right)
$$

where $C$ is a consumption aggregate. The utility function has standard properties.

Production of $X$ and $Y$ satisfies:

$$
X=F\left(A_{x}, L_{x}, K_{x}\right), \quad Y=G\left(A_{y}, L_{y}, K_{y}\right)
$$

where $F$ and $G$ have standard properties. $L_{i}, K_{i}, i=x, y$ are the amounts of labor and capital employed in the $X$ and $Y$ sectors, respectively. $A_{x}$ and $A_{y}$ are random variables representing variation in the state of technology in these two sectors.

Factor allocations satisfy:

$$
\begin{aligned}
L_{x}+L_{y} & =L \\
K_{x}+K_{y} & =K,
\end{aligned}
$$

where $K$ and $L$ are fixed.

Let $s_{l x} \equiv L_{x} / L, s_{k x} \equiv K_{x} / K$. A social planner selects $\left\{s_{l x}, s_{k x}\right\}$ in order to maximize (1) subject to (2)-(4). In order to examine the effects of uncertainty, we first need to know the allocations under certainty. For now, let $A_{x}=A_{y}=A$. First order conditions (FOCs) are:

$$
\begin{aligned}
& \frac{d C}{d c_{x}} \frac{d c_{x}}{d s_{l x}}-\frac{d C}{d c_{y}} \frac{d c_{y}}{d s_{l y}}=0 \\
& \frac{d C}{d c_{x}} \frac{d c_{x}}{d s_{k x}}-\frac{d C}{d c_{y}} \frac{d c_{y}}{d s_{k y}}=0
\end{aligned}
$$

where $s_{l y}=1-s_{l x}$ and $s_{k y}=1-s_{k x}$. Equations (5)-(6), together with the production functions and the equilibrium relations $c_{x}=X$ and $c_{y}=Y$, determine the optimal levels of $X$ and $Y$ under certainty, $\bar{X}$ and $\bar{Y}$.

\section{Uncertainty}

We now introduce uncertainty. We assume that the production decisions, namely the allocation of labor and capital across sectors by the social planner, are made before the resolution 
of uncertainty. Once the sectorial allocations of labor and capital have been determined, uncertainty is resolved and production ensues. After that, consumption takes place.

The optimality conditions now take the form:

$$
\begin{gathered}
E \frac{d U}{d C}\left(\frac{d C}{d c_{x}} \frac{d c_{x}}{d s_{l x}}-\frac{d C}{d c_{y}} \frac{d c_{y}}{d s_{l y}}\right)=0 \\
E \frac{d U}{d C}\left(\frac{d C}{d c_{x}} \frac{d c_{x}}{d s_{k x}}-\frac{d C}{d c_{y}} \frac{d c_{y}}{d s_{k y}}\right)=0
\end{gathered}
$$

where $E$ denotes the expectations operator with respect to the distribution of output shocks, $A_{x}$ and $A_{y}$.

If the term inside the parenthesis does not contain aggregate consumption, then the FOCs are identical under certainty and uncertainty. For instance, this would be the case if the consumption aggregate, $C$, were logarithmic. In general, more structure needs to be imposed in order to determine the effects of uncertainty. We consider a Constant Elasticity of Substitution (CES) specification for utility and production.

The utility function is:

$$
\begin{gathered}
U=\frac{1}{1-\gamma} C^{1-\gamma}=\frac{1}{1-\gamma}\left[\left(w_{c} c_{x}^{\rho}+\left(1-w_{c}\right) c_{y}^{\rho}\right)^{\frac{1}{\rho}}\right]^{1-\gamma}, \\
\rho \in(-\infty, 1], \gamma \geq 0,0<w_{c}<1
\end{gathered}
$$

and the production functions are:

$$
\begin{aligned}
c_{x}=X & =A_{x}\left[w_{x}\left(s_{x} L\right)^{\theta}+\left(1-w_{x}\right)\left(s_{k x} K\right)^{\theta}\right]^{\frac{1}{\theta}} \\
c_{y}=Y & =A_{y}\left[w_{y}\left(s_{l y} L\right)^{\theta}+\left(1-w_{y}\right)\left(s_{k y} K\right)^{\theta}\right]^{\frac{1}{\theta}} \\
\theta & \in(-\infty, 1], 0<w_{i}<1, i=x, y .
\end{aligned}
$$

Equations (7)-(8) now take the form:

$$
\begin{aligned}
E U_{s_{l x}} & =E\left\{C^{1-\gamma-\rho}\left(A_{x}^{\theta} c_{x}^{\rho-\theta} w_{c} w_{x} s_{l x}^{\theta-1}-A_{y}^{\theta} c_{y}^{\rho-\theta}\left(1-w_{c}\right) w_{y} s_{l y}^{\theta-1}\right)\right\} \\
& =E\left(U_{s_{l x}}\left(s_{l x}, s_{k x}, A_{x}, A_{y}\right)\right)=0 \\
E U_{s_{k x}}=E\left\{C^{1-\gamma-\rho}\left(A_{x}^{\theta} c_{x}^{\rho-\theta} w_{c}\left(1-w_{x}\right) s_{k x}^{\theta-1}-A_{y}^{\theta} c_{y}^{\rho-\theta}\left(1-w_{c}\right)\left(1-w_{y}\right) s_{k y}^{\theta-1}\right)\right\} & =E\left(U_{s_{k x}}\left(s_{l x}, s_{k x}, A_{x}, A_{y}\right)\right)=0
\end{aligned}
$$


Note that the fact that $s_{l x}$ and $s_{k x}$ are selected (known) before the resolution of uncertainty implies that these two first-order conditions can be combined to give:

$$
\frac{w_{x}}{1-w_{x}}\left(\frac{s_{l x}}{s_{k x}}\right)^{\theta-1}=\frac{w_{y}}{1-w_{y}}\left(\frac{s_{l y}}{s_{k y}}\right)^{\theta-1} .
$$

Therefore, $s_{k x}$ is a deterministic function of $s_{l x}$ and knowledge of the latter suffices to characterize the behavior of the model under uncertainty.

If the elasticity of substitution in consumption, $\rho$, is equal to zero (a logarithmic consumption aggregate, $C$ ) then the terms inside the parenthesis in (11) and (12) are independent of $A_{x}$ and $A_{y}$. It follows immediately that uncertainty does not matter for the optimal allocation of resources.

The situation is more complicated when $\rho \neq 0$. In this case, the effects of uncertainty can be determined using the Hahn-Rothschild-Stiglitz approach. Suppose that we want to maximize $E U(s, A)$ with regard to $s$, where $A$ is a random variable. The first order condition is $E U_{s}(s, A)=0$ and the second order condition is $E U_{s s}(s, A)<0$. The Hahn-RothschildStiglitz theorem (see Levhari (1972)) states that, if $E U_{s}(s, A)$ is a concave function of $A$, then higher uncertainty (a mean preserving spread in $A$ ) decreases the optimal choice of $s$. If it is a convex function, then $s$ increases with higher uncertainty.

We thus need to determine the curvature of the term inside the expectation in (11), $U_{s_{l x}}\left(s_{l x}, A_{x}, A_{y}\right)$. Without loss of generality we assume that only sector $X$ is subject to uncertainty and we set $A_{y}=1$. The sign of the second derivative of $U_{s_{l x}}\left(s_{l x}, A_{x}, A_{y}\right)$ with regard to $A_{x}$, evaluated at the deterministic steady state, is $U_{A_{x} A_{x}}^{s_{l x}}$,

$$
\operatorname{sign}\left(U_{A_{x} A_{x}}^{s_{l x}}\right)=\operatorname{sign}\{\rho(1-\rho)(\psi-1)-\gamma \rho \psi\},
$$

where $\psi=2 w_{c}\left(\frac{c_{x}}{C}\right)^{\rho}$, that is, two times the expenditure share of good 1 in total consumption ${ }^{3}$, or, equivalently, its share in GDP. If $U_{A_{x} A_{x}}^{s_{l x}}>0(<0)$ then higher uncertainty in sector X attracts (repels) resources.

For $\rho \neq 0$ or $\rho \neq 1$, the sign of the effects of uncertainty depends on the location of the deterministic equilibrium on the production possibilities frontier through the $\psi$ term. If $\psi \leq 0.5$ then (14) takes the simpler form

$$
\operatorname{sign}\left(U_{A_{x} A_{x}}^{s_{l x}}\right)=-\operatorname{sign}\{\rho\}
$$

\footnotetext{
${ }^{3}$ This follows from the maximization of $P C-p_{x} c_{x}+p_{y} c_{y}$ with regard to $c_{x} \cdot p_{x}$ and $p_{y}$ are the prices of $\mathrm{X}$ and $\mathrm{Y}$ respectively and $\mathrm{P}$ is the $\mathrm{CPI}$.
} 
In order to gain some intuition on the determinants of the effects of uncertainty, it may be useful to think in terms of expected excess returns. Let us define the marginal excess return -in terms of aggregate consumption- in sector $\mathrm{X}$ as $M E R$,

$$
M E R=\frac{d C}{d c_{x}} \frac{d c_{x}}{d s_{l x}}-\frac{d C}{d c_{y}} \frac{d c_{y}}{d s_{l y}}
$$

$M E R$ indicates how much extra total consumption $\mathrm{C}$ will be generated by moving one unit of labor from sector $\mathrm{Y}$ to sector $\mathrm{X}$. This depends on how much extra $\mathrm{X}$ and less $\mathrm{Y}$ are produced as a result of this relocation, which in turn depends on the slope of the production possibilities frontier ${ }^{4}$ and on how much these changes in $\mathrm{X}$ and $\mathrm{Y}$ affect aggregate consumption, which depends on the slope of the consumption possibilities frontier ${ }^{5}$.

The curvature of $M E R$ as a function of uncertainty, $A_{x}$, plays a critical role in the determination of the effects of uncertainty on the optimal allocation of resources. The sign of the curvature is determined by

$$
\operatorname{sign}\left\{\frac{d^{2} M E R}{d A_{x}^{2}}\right\}=\operatorname{sign}\{\rho(1-\rho)(\psi-1)\}
$$

Note that this is simply the first term in expression (14). Let us try to understand its role. If $M E R$ is a convex function of $A_{x}$, then the expected $M E R$ is positive when evaluated at the deterministic equilibrium. Hence, in the neighborhood of this equilibrium, relocating an extra unit of labor from sector $\mathrm{Y}$ to $\mathrm{X}$ increases the mean of aggregate consumption, $C$. The reverse pattern obtains if $M E R$ is concave. The positive excess return in the risky sector serves as an attractor for that sector. Nonetheless, the total effect (the sign of (14)) cannot be determined without also taking into account the variability of the excess return (which changes as a result of a relocation across sectors) - as well as the degree of aversion that agents have towards consumption variability. These considerations operate through the last term in expression (14) which contains the degree of aversion to variability in both total consumption $(\gamma)$ and its composition $(\rho)$. When $\rho<0$ and $\psi<0.5$ then moving resources to the risky sector not only generates a positive excess return but it also provides insurance ${ }^{6}$.

\footnotetext{
${ }^{4}$ The production possibilities frontier is defined the standard way. Namely, as $F(X, Y)$ where $\mathrm{X}$ and $\mathrm{Y}$ satisfy the first order conditions of the profit maximization problem of the firms.

${ }^{5}$ The consumption possibilities frontier is defined in a way analogous to the production possibilities frontier. Namely as $G\left(c_{x}, c_{y}\right)$, where $c_{x}$ and $c_{y}$ satisfy the first order conditions of the household.

${ }^{6}$ When the two goods are poor substitutes $(\rho<0)$, then individual good consumption smoothing becomes important relative to the smoothing of the aggregate bundle. Low realizations of the stochastic output are costly, in particular when its expenditure share is less than $50 \%$. The agents attempt to get insurance against this by making a bigger investment in the sector facing uncertainty. Thinking about the extreme case of a Leontief production function can help understand this point better.
} 
When $\rho>0$, a positive expected marginal excess return may not suffice to draw additional resources because the individuals are concerned more about variability in total consumption - which rises when more resources are devoted to the risky sector - than variability in its composition $^{7}$.

Naturally, if the agents do not care about variability in total consumption $(\gamma=0)$ then a positive $M E R$ will always attract resources into the risky sector.

Uncertainty in both sectors The case of uncertainty in both sectors can be treated in a manner analogous to that of the previous section. Unfortunately, the approximated version of the optimality condition is not particularly revealing for gaining any insights into the effects of uncertainty on the pattern of production. ${ }^{8}$

Nonetheless, there is a special case of both theoretical and practical importance, that can easily be studied. Namely, the case of aggregate uncertainty, where shocks to both sectors are perfectly correlated. It can be easily verified that in this case the allocations under uncertainty are identical to those under certainty.

\section{Conclusions}

We have analyzed the effects of uncertainty on the optimal allocation of resources in a general equilibrium, two-good, two-factor model with complete asset markets. This task has been long overdue as, surprisingly, there exists no treatment of this issue in the literature.

The level of flexibility (elasticity of substitution) in consumption turns out to play the key role. Risk aversion may also matter but in a more limited capacity while production structure does not seem to play any direct role. Risk aversion is not necessary as uncertainty influences the allocation of resources even under risk neutrality. A key implication of the analysis is that the lack of consumption flexibility encourages the taking of more risk when the risky sectors do not make up too large a share of GDP. To the extent that activities with greater uncertainty also carry greater average returns, economies with limited substitutability in consumption will tend to outperform the more flexible economies.

\footnotetext{
${ }^{7}$ Recall that the greater the degree of substitutability between $X$ and $Y$, the greater the dependence of the marginal utility of $X$ and $Y$ on their sum, $X+Y$, rather than on $X$ and $Y$ individually

${ }^{8}$ It should be kept in mind that, for any parametrization of the model, it is always feasible to also solve the model numerically and derive the sectorial factor shares and levels of output.
} 


\section{References}

Helpman, E. and A. Razin (1978) A theory of international trade under uncertainty, New York: Academic Press.

King, R. and S. Rebelo (1999) "Resuscitating real business cycles" Handbook of macroeconomics, Volume 1B, 1999, pp. 927-1007. New York: Elsevier Science, North-Holland.

Kydland, F. and E. Prescott (1982) "Time to build and aggregate Fluctuations" Econometrica, 50, 6, 1345-70.

Levhari, D. (1972) "Optimal savings and portfolio choice under uncertainty", in Szego and Shell (eds), Mathematical methods in investment and finance, North Holland.

Obstfeld, M. (1994) "Risk-Taking, global diversification, and growth" American Economic Review, v. 84, 5, 1310-29.

Rothemberg, T., and K. Smith (1971) "The effect of uncertainty on the resource allocation in a general equilibrium model" Quarterly Journal of Economics, 85(3), 440-454. 